\title{
GMR
}

\section{Is differential expression of p16INK4a based on the classification of uterine smooth muscle tumors associated with a different prognosis? A meta-analysis}

\author{
H.Y. Cao ${ }^{1 *}$, S. Yang ${ }^{2 *}$, S. Wang ${ }^{3}$, L.Y. Deng ${ }^{3}$ and J.Y. Lou ${ }^{1}$ \\ ${ }^{1}$ Department of Gynecology and Obstetrics, \\ West China Second University Hospital, Sichuan University, \\ Chengdu, Sichuan Province, China \\ ${ }^{2}$ Laboratory of Lung Development and Disease West China Second University \\ Hospital, Sichuan University, Chengdu, Sichuan Province, China \\ ${ }^{3}$ Key Laboratory of Birth and Related Diseases of Women and Children, \\ Sichuan University Ministry of Education, Chengdu, Sichuan Province, China \\ *These authors contributed equally to this study. \\ Corresponding author: J.Y. Lou \\ E-mail: loujiangyan2@163.com
}

Genet. Mol. Res. 16 (1): gmr16019481

Received October 27, 2016

Accepted December 5, 2016

Published March 22, 2017

DOI http://dx.doi.org/10.4238/gmr16019481

Copyright (C) 2017 The Authors. This is an open-access article distributed under the terms of the Creative Commons Attribution ShareAlike (CC BY-SA) 4.0 License.

\begin{abstract}
We conducted a meta-analysis to examine p16INK4a expression in uterine smooth muscle tumors (USMTs). Although the prognostic value of tumor suppressor p16INK4a has been elucidated in a variety of cancers and precancerous lesions, its role in USMTs is not well established. We searched PubMed, Web of Science, and Embase for publication son p16INK4a expression in USMTs. Strict inclusion and exclusion criteria were imposed. Risk ratios (RRs) with $95 \%$ confidence intervals $(95 \% \mathrm{CIs})$ were calculated to assess the strength of association. Publication bias was estimated using funnel plots and
\end{abstract}


the Egger's regression test. Twelve eligible studies comprising 661 patients were included. Compared with leiomyoma (LM), the figures for the strength of association were as follows: $\mathrm{LM}$ variants $(\mathrm{RR}=1.53$, $95 \% \mathrm{CI}=1.03-2.27, \mathrm{P}=0.036$, random effect); leiomyosarcoma (LMS) $(\mathrm{RR}=3.20,95 \% \mathrm{CI}=1.68-6.12, \mathrm{P}<0.001$, random effect $)$; and smooth muscle tumors of uncertain malignant potential (STUMP) $(\mathrm{RR}=2.90$, $95 \% \mathrm{CI}=1.17-7.21, \mathrm{P}=0.022$, random effect). $\mathrm{p} 16 \mathrm{INK} 4 \mathrm{a}$ expression was significantly higher in LMS than in LM variants $(\mathrm{RR}=3.74,95 \% \mathrm{CI}$ $=1.96-7.13, \mathrm{P}<0.001$, random effect $)$ or STUMP $(\mathrm{RR}=1.67,95 \% \mathrm{CI}$ $=1.26-2.23, \mathrm{P}<0.001$, fixed effect). There was a significant correlation between overexpressed p16INK4a and recurrence rates of USMTs (RR $=1.85,95 \% \mathrm{CI}=1.11-3.10, \mathrm{P}=0.019$, fixed effect). p16INK4a over expression is a potential biomarker for diagnosing problematic USMTs and it might indicate a worse prognosis. However, there is currently insufficient evidence to assess the prognostic value of p16INK4a in USMTs.

Key words: p16INK4a; Leiomyoma; Leiomyosarcoma; Meta-analysis

\section{INTRODUCTION}

Uterine smooth muscle tumors (USMTs) are the most common neoplasm in the female reproductive system. Usual leiomyoma (UM) is the most common benign tumor in the uterus, whereas malignant leiomyosarcoma (LMS) accounts for only $2.3 \%$ of uterine neoplasms (Boll et al., 2012). There are also leiomyoma (LM) variants (mitotically active, cellular, and atypical leiomyomas) that are characterized by mostly benign behavior. Insome respects, LM variants have morphological similarities with LMS such as coagulative tumor cell necrosis, and the degree of cytologic atypia or mitotic activity. Finally, there are smooth muscle tumors of uncertain malignant potential (STUMP), which cannot be classified as unequivocally benign or malignant (Tavassoli and Devilee, 2003). Because of this wide range of subtypes and overlapping features, diagnostic problems still exist, despite differential diagnoses based on histopathological features, making clinical management difficult.

p16INK4a protein, encoded by the INK4A locus, inactivates the cell cycle by inhibiting cyclin-dependent kinases (CDKs) (Ruas and Peters,1998). Loss ofp16INK4a expression seems to be associated with the development and progression of cancers. The authors of previous studies have reported the diagnostic and prognostic value of overexpressed p16INK4a protein in various cancers and premalignant lesions (Sano et al., 1998; Bu et al., 2014; Huang et al., 2014; Cao et al., 2016). Although p16INK4aoverexpression might be used as a surrogate marker for human papilloma virus (HPV) in cervical and vaginal carcinoma, its overexpression in other carcinomas is not necessarily related to HPV (Hellman et al., 2014). However, its diagnostic and prognostic role in USMTs remains ambiguous (Atkins et al., 2008; Mills et al., 2013). Therefore, in the present meta-analysis, which had aPICOS (population, intervention, comparators, outcomes, study) structure, we aimed to compare the level of p16INK4a expression between patients with different types of USMTs and investigate its potential prognostic value.

Genetics and Molecular Research 16 (1): gmr16019481 


\section{METHODS}

\section{Search strategy}

We searched PubMed, Web of Science, and Embase databases between January 1991 and September 2016to identify all articles that investigated the association between p16INK4a expression and USMTs. All relevant articles were retrieved using the following search terms and their combinations: "p16 or p16INK4a" and "uterine leiomyoma", "uterine sarcoma", "uterine leiomyosarcoma", "uterine leiomyoma variants", "smooth muscle tumors of uncertain malignant potential", or "STUMP". References in the identified publications were also screened for other relevant studies. For multiple publications including overlapping data, only the newest or largest-scale study was included. Two independent investigators first searched potentially relevant studies by screening the title and abstract, and then browsed the full texts. Any discrepancy was resolved by discussion.

\section{Inclusion criteria}

We included the following: studies in which the diagnosis of USMTs had been proven by pathological methods; studies of p16INK4a expression that were based on USMT tissue; specimens examined by immunohistochemistry; and all studies on the correlation of p16INK4a expression with USMT tissue and the association of p16INK4a overexpression with overall survival (OS) or disease-free survival (DFS), or recurrence of USM Tin patients. No limitation was set on the minimum number of patients in each study for inclusion in the analysis.

\section{Exclusion criteria}

We excluded the following: review articles without original data; studies in languages other than English; studies based on serum or any other kinds of specimen; and studies using methods other than immunohistochemistry to examine specimens.

\section{Data extraction and quality assessment}

Three investigators (Hanyu Cao, Si Wang, and Liyun Deng) extracted information independently from all articles according to the inclusion criteria listed above. Any dispute was resolved by discussion until a consensus was reached between the investigators. The following information was collected from each publication: the first author's name, the publication year, the patient's country, the technique used, the percentage of p16INK4a-positive cells in each subtype of USMT, the number of patients, and the cut-off value of overexpression of p16INK4ain the included studies. In addition, instead of using a quality-related score system, we controlled the study quality by weighting each study with strict inclusion or exclusion criteria because there was no agreement for a standardized score system for observational studies.

\section{Statistical analysis}

The cut-off value of over expressed p16INK4a according to the stained cells is presented in Table 1 for each study. Risk ratios (RR) and 95\% confidence intervals (95\%CIs)

Genetics and Molecular Research 16 (1): gmr16019481 
were pooled to explore whether there was a difference in p16INK4a expression between these various groups of USMTs and to evaluate the association between p16INK4a expression and patients' survival. Heterogeneity was investigated using Cochran's chi-square Q test with a significance level of $\mathrm{P}<0.10$ and an $\mathrm{I}^{2}>50 \%$, the latter value representing the proportion of total variation contributed by between-study variations. In such cases, the random-effects model was applied to estimate the pooled RRs (DerSimonian and Kacker, 2007). Otherwise, the pooled RRs were estimated by the fixed-effects model. In addition, funnel plots and the Egger's linear regression test were used to investigate publication bias (Egger and Smith, 1998). All statistical tests were carried out using Stata 12.0 software (Stata Corp, USA).

\begin{tabular}{|c|c|c|c|c|c|c|c|c|c|}
\hline Study & Year & Patient's country & Technique & LM & LM variants & LMS & STUMP & Number of patients & Cut-off (IHC) \\
\hline Bodner-Adler & 2005 & Austria & $\mathrm{IHC}$ & $3 / 26$ & ND & $12 / 21$ & $5 / 24$ & 71 & $33 \%$ \\
\hline O'Neill & 2007 & UK & IHC & $0 / 10$ & $7 / 27$ & $19 / 22$ & $1 / 4$ & 63 & $5 \%$ \\
\hline Gannon & 2008 & Canada & $\mathrm{IHC}$ & $3 / 12$ & $9 / 35$ & $8 / 8$ & ND & 66 & Any moderate/strong \\
\hline Chen & 2008 & USA & IHC & $5 / 35$ & $18 / 28$ & $35 / 35$ & $2 / 2$ & 100 & $25 \%$ \\
\hline Atkins & 2008 & USA & IHC & $11 / 15$ & ND & ND & $3 / 8$ & 46 & $66 \%$ \\
\hline IP & 2009 & China and Canada & IHC & $\mathrm{ND}$ & ND & ND & $2 / 15$ & 16 & $66 \%$ \\
\hline Yanai & 2010 & Japan & IHC & $1 / 4$ & ND & $2 / 4$ & ND & 4 & $75 \%$ \\
\hline Ünver & 2011 & Turkey & IHC & $0 / 15$ & $1 / 14$ & $17 / 21$ & $1 / 3$ & 53 & $10 \%$ \\
\hline Hakverdi & 2011 & Turkey & IHC & $0 / 6$ & $0 / 9$ & $5 / 15$ & ND & 40 & $66 \%$ \\
\hline Mills & 2013 & USA & $\mathrm{IHC}$ & ND & $14 / 52$ & $11 / 16$ & ND & 68 & $66 \%$ \\
\hline Slatter & 2015 & China and New Zealand & IHC & ND & ND & $14 / 24$ & $6 / 15$ & 72 & $70 \%$ \\
\hline Liang & 2015 & China & IHC & $0 / 15$ & $4 / 32$ & $7 / 15$ & ND & 62 & $75 \%$ \\
\hline
\end{tabular}

$\mathrm{IHC}=$ immunohistochemistry; $\mathrm{LM}$ variants = leiomyoma variants; $\mathrm{LMS}=$ leiomyosarcoma ND = no data; STUMP $=$ smooth muscle tumors of uncertain malignant potential.

\section{RESULTS}

\section{Study inclusion and characteristics}

As shown in Figure 1, a total of 12 eligible studies comprising 661 patients from different countries were included in this meta-analysis, with the number of patients ranging from 4 to 100 per study (Bodner-Adler et al., 2005; O'Neill et al., 2007; Atkins et al., 2008; Chen and Yang, 2008; Gannon et al., 2008; Ip et al., 2009; Yanai et al., 2010; Hakverdi et al., 2011; Ünver et al., 2011; Mills et al., 2013; Liang et al., 2015; Slatter et al., 2015). Nine of the studies investigated P16INK4a expression in LM, seven presented data on LM variants, ten presented data on LMS, and seven studies reported STUMP. Moreover, only four studies provided sufficient data on the association betweenp16INK4a and survival data. All the studies used the immune histochemical method with different cut-off values. The main characteristics of these studies are summarized in Table 1.

\section{Differential expression of p16INK4ain USMTs}

As shown in Figure 2, the overexpression of p16INK4a was significantly associated with $\mathrm{LM}$ variants $(\mathrm{RR}=1.53,95 \% \mathrm{CI}=1.03-2.27, \mathrm{P}=0.036$, random effect; Figure $2 \mathrm{~A})$, LMS $(\mathrm{RR}=3.20,95 \% \mathrm{CI}=1.68-6.12, \mathrm{P}<0.001$, random effect; Figure $2 \mathrm{~B})$, and STUMP $(\mathrm{RR}=$ $2.90,95 \% \mathrm{CI}=1.17-7.21, \mathrm{P}=0.022$, random effect; Figure $2 \mathrm{C}$ ), compared with LM. There was a significantly higher level of p16INK4a expression in LMS than in LM variants (RR $=3.74,95 \% \mathrm{CI}=1.96-7.13, \mathrm{P}<0.001$, random effect; Figure $2 \mathrm{D})$, or STUMP $(\mathrm{RR}=1.67$, 
$95 \% \mathrm{CI}=1.26-2.23, \mathrm{P}<0.001$, fixed effect; Figure 2E). However, the difference in p16INK4a expression between STUMP and LM variants was not statistically significant $(\mathrm{RR}=1.86$, $95 \% \mathrm{CI}=0.50-6.93, \mathrm{P}=0.357$, fixed effect; Figure $2 \mathrm{~F}$ ).

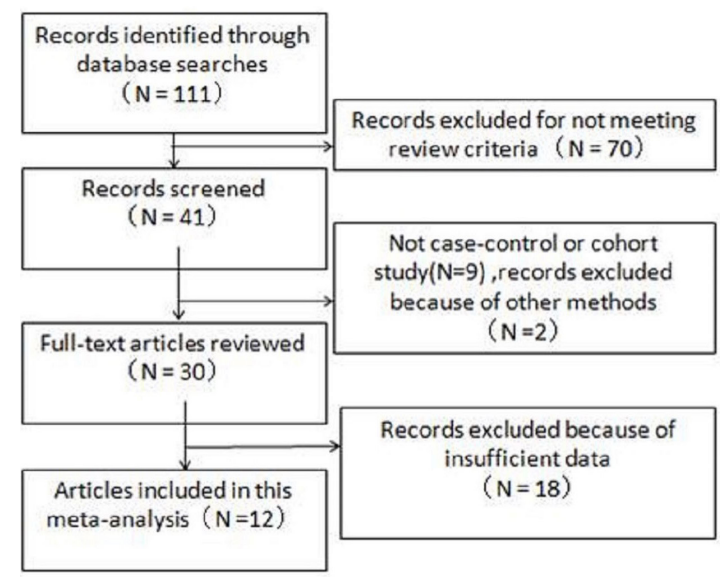

Figure 1. Flow chart demonstrating studies processed for inclusion in the meta-analysis.

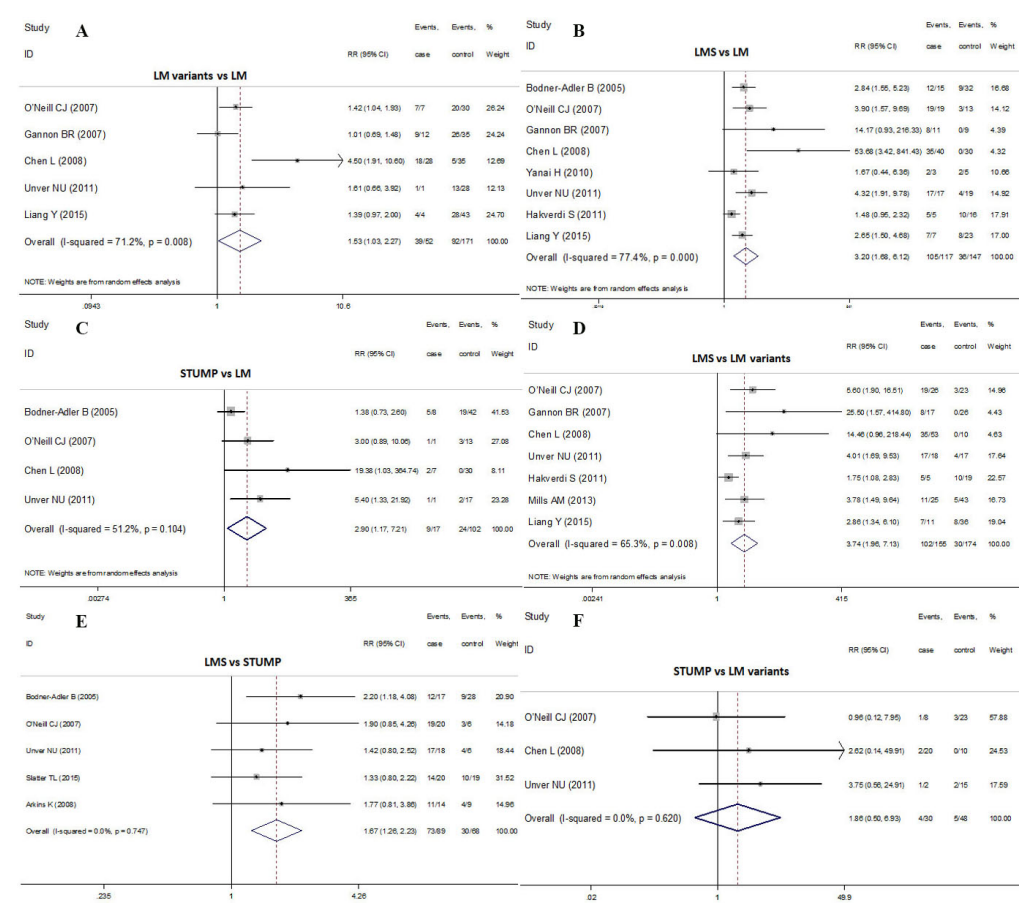

Figure 2.Forest plots of the association between p16INK4a overexpression in leiomyoma (LM) vs LM variants (A); leiomyosarcoma (LMS) vs leiomyoma (LM) variants (B); smooth muscle tumors of uncertain malignant potential (STUMP) vs leiomyoma (LM) (C); leiomyosarcoma (LMS) vs leiomyoma (LM) variants (D); leiomyosarcoma (LMS) vs smooth muscle tumors of uncertain malignant potential (STUMP) (E); and smooth muscle tumors of uncertain malignant potential (STUMP) vs leiomyoma (LM) variants (F).

Genetics and Molecular Research 16 (1): gmr16019481 


\section{Correlation between p16INK4a expression and recurrence rates}

Recurrence rates were extracted from four studies and were pooled according to the classification of USMTs using the method mentioned earlier. The association between overexpressed p16INK4aand the recurrence rates of USMTs was statistically significant $(\mathrm{RR}=$ $1.85,95 \% \mathrm{CI}=1.11-3.10, \mathrm{P}=0.019$, fixed effect; Figure 3 ). Subgroup analysis showed that only STUMP $(\mathrm{RR}=3.34,95 \% \mathrm{CI}=1.23-9.07, \mathrm{P}=0.018$, fixed effect; Figure 3 ) was significantly correlated with P16INK4a, and no significant association was found between P16INK4a and LMS $(\mathrm{RR}=1.57,95 \% \mathrm{CI}=0.85-2.90, \mathrm{P}=0.148$, fixed effect; Figure 3$)$ or $\mathrm{LM}$ variants.

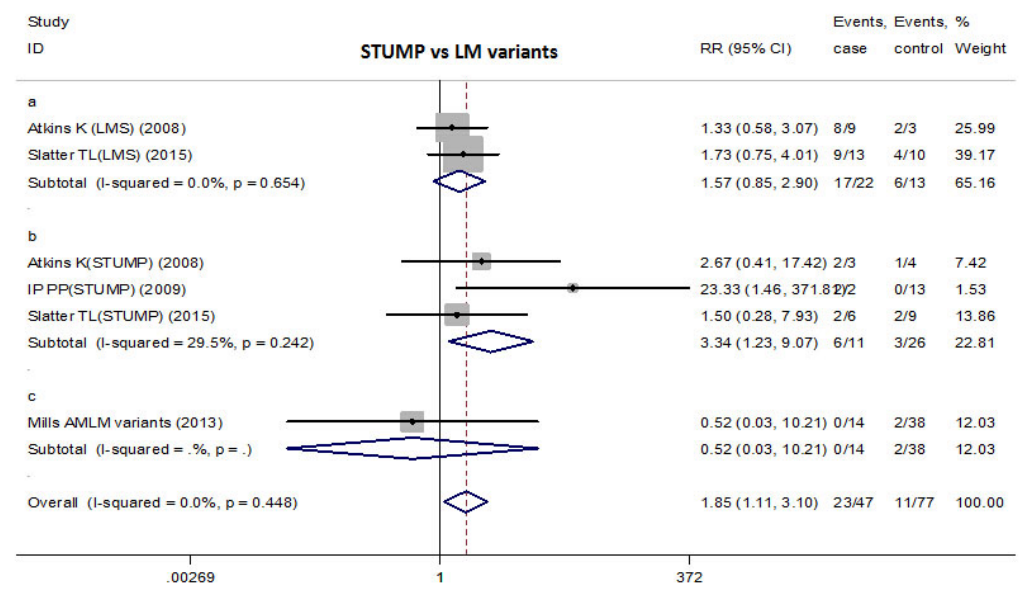

Figure 3. Forest plot for the association between p16INK4a expression and recurrence rates.

\section{Publication bias}

A Begg's funnel plot and the Egger's test were applied to recurrence rates to determine whether publication bias existed in the studies. The shape of the funnel plot did not show obvious asymmetry. No obvious evidence of asymmetry was found in the Egger's test of recurrence rates $(\mathrm{P}=0.56$; Figure 4$)$.

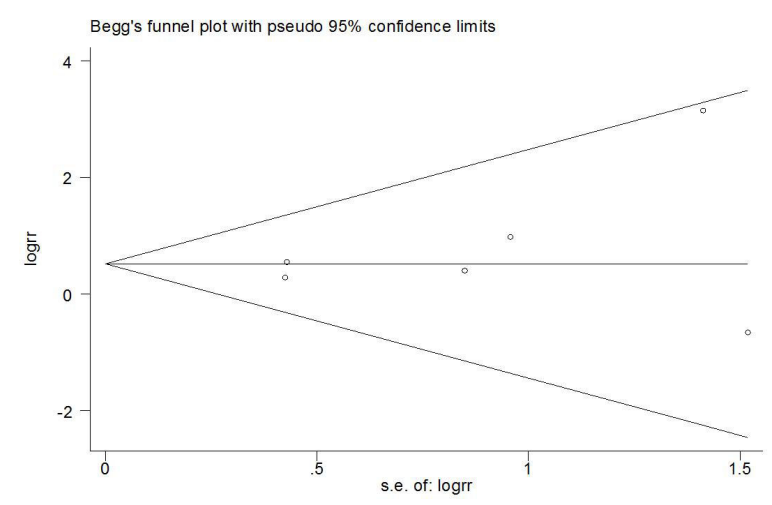

Figure 4. Funnel plot analysis on the detection of publication bias in the meta-analysis of the prognostic value of p16INK4a in the recurrence rates of uterine smooth muscle tumors (USMTs).

Genetics and Molecular Research 16 (1): gmr16019481 


\section{DISCUSSION}

$\mathrm{P} 16 \mathrm{INK} 4 \mathrm{a}$ is a classic tumor suppressor that is encoded by the $C D K N 2 A$ gene. It is able to inactivate the retinoblastoma (RB1) family of tumor suppressor proteins by inhibiting cyclindependent kinases CDK4 and CDK6, leading to cell-cycle arrest (Ortega et al., 2002). Various human cancers are characterized by the functional loss of the INK4a family of proteins and the over expression of p16INK4a. Such factors are significantly associated with the outcome of many malignant tumors such as cervical cancer, osteosarcoma, gastrointestinal stromal tumor, head and neck squamous cell carcinoma, and non-small lung cancer (Zong et al., 2012; Bu et al., 2014; Huang et al., 2014; Ndiaye et al., 2014; Huang et al., 2015). There remains a scarcity of published evidence to support the role of over expressed p16INK4a in USMTs.

Uterine LM and LMS are at opposite ends of the pathological spectrum of uterine smooth muscle tumors, with several LM variants and STUMP in between (Arleo et al., 2015). LM occurs in nearly $80 \%$ of women of reproductive age, although only $30 \%$ experience symptoms that are so severe that they are compelled to seek treatment (Moravek et al., 2015). Moreover, the identification of LM variants and STUMP currently hampers the diagnosis of USMTs, although the 2003 World Health Organization (WHO) diagnostic criteria for USMTs has distinguished some rare variants of leiomyoma (Hendrickson et al., 2003). LMS is the most malignant USMT; it is associated with a high risk of recurrence and metastasis, and a poor overall survival rate of only $10-25 \%$ (Zaloudek and Hendrickson, 2002). LMS is mainly treated by surgery that comprises total abdominal hysterectomy and bilateral salpingooophorectomy. No convincing evidence for the effectiveness of adjuvant radiation therapy or chemotherapy has been reported. However, some times, histopathological examination reveals that a presumptive LM uterine tumor is actually an LMS or an LM variant. This is because in many cases, imaging examination reveals no obvious signs, and no typical clinical manifestations are presented. Therefore, there is a pressing need to discover biomarkers for the early differential diagnosis of USMTs and indicators for prognosis that provide guidance for early treatment and clinical management. This study is possibly the first meta-analysis that has evaluated the diagnostic and prognostic value of p16INK4a in USMTs.

The present meta-analysis using pooled RRs demonstrated that p16INK4aover expression correlates significantly with LMS, STUMP, and LM variants compared with LM. The classification of STUMP and LM variants is problematico wing to their unpredictable clinical course and the lack of clinical evidence (Peters et al., 1994). Atkins et al. (2008) suggested that STUMPs should be classified as LMSs, and Ip et al. (2009) and Yanai et al., (2010) concluded that patients diagnosed with STUMPs should receive long-term surveillance owing to their often delayed recurrence and low-grade malignancy behavior. However, all the patients with STUMPs in the study by Bodner-Adler et al. (2005) had favorable outcomes.

Although many previous studies have reported p16 to be a useful marker for the discrimination of LMS from other types of USMT, the difference is not very pronounced in the study by Atkins et al. (2008). Moreover, very few studies presented a recurrence rate related to p16INK4aand USMTs after repeated searches, which may have been due to the low frequency of malignant USMTs. Analysis of outcome in the present study revealed a statistically significant connection between p16INK4a overexpression and recurrence rates of USMTs, in accordance with the study by D'Angelo et al. (2011), who reported that the immunoreactions of p16 had a negative effect on disease-free survival. Both Ip et al. (2009) and Atkins et al. (2008) concluded that strong, diffuse p16 expression might help to identify the

Genetics and Molecular Research 16 (1): gmr16019481 
recurrence of STUMPs, whereas Mills et al. (2013) denied the prognostic value of p16INK4a in atypical LMs.

Nevertheless, this study had some limitations. First, it had a small sample size of 621 patients, which may not have provided sufficient statistical evidence to prove the role of p16INK4a in USMTs. Studies related to the survival data for USMTs are scarce. Second, to a certain extent, the treatment for each patient varied, depending on the particular clinical manifestations, which should not be ignored. Third, the lack of a standardized immunostaining point of cut-off for positive p16INK4a expression may have led to inaccurate results and variations in different studies. For these reasons, more large-scale studies and a greater body of evidence are needed to investigate p16INK4a expression in USMTs.

In conclusion, despite its limitations, the present meta-analysis suggests that overexpressed p16INK4a correlates significantly with LMS and LM variants compared with LM, and there is a higher level of p16INK4a expression in LMS than in LM variants. The assessment of p16INK4a expression is capable of providing better diagnostic information for patients with USMTs. Further studies are needed to verify this conclusion and to identify the prognostic value of p16INK4a expression in USMTs.

\section{Conflicts of interest}

The authors declare no conflict of interests.

\section{REFERENCES}

Arleo EK, Schwartz PE, Hui P and McCarthy S (2015). Review of leiomyoma variants. AJR Am. J. Roentgenol. 205: 912921. http://dx.doi.org/10.2214/AJR.14.13946

Atkins KA, Arronte N, Darus CJ and Rice LW (2008). The Use of p16 in enhancing the histologic classification of uterine smooth muscle tumors. Am. J. Surg. Pathol. 32: 98-102. http://dx.doi.org/10.1097/PAS.0b013e3181574d1e

Bodner-Adler B, Bodner K, Czerwenka K, Kimberger O, et al. (2005). Expression of p16 protein in patients with uterine smooth muscle tumors: an immunohistochemical analysis. Gynecol. Oncol. 96: 62-66. http://dx.doi.org/10.1016/j. ygyno.2004.09.026

Boll D, Verhoeven RH, van der Aa MA, Pauwels P, et al. (2012). Incidence and survival trends of uncommon corpus uteri malignancies in the Netherlands, 1989-2008. Int. J. Gynecol. Cancer 22: 599-606. http://dx.doi.org/10.1097/ $\underline{\text { IGC.0b013e } 318244 \mathrm{cedc}}$

Bu J, Li H, Liu LH, Ouyang YR, et al. (2014). P16INK4a overexpression and survival in osteosarcoma patients: a meta analysis. Int. J. Clin. Exp. Pathol. 7: 6091-6096.

Cao H, Wang S, Zhang Z and Lou J (2016). Prognostic value of overexpressed p16INK4a in vulvar cancer: a metaanalysis. PLoS One 11: e0152459. http://dx.doi.org/10.1371/journal.pone.0152459

Chen L and Yang B (2008). Immunohistochemical analysis of p16, p53, and Ki-67 expression in uterine smooth muscle tumors. Int. J. Gynecol. Pathol. 27: 326-332. http://dx.doi.org/10.1097/PGP.0b013e31815ea7f5

D’Angelo E, Espinosa I, Ali R, Gilks CB, et al. (2011). Uterine leiomyosarcomas: tumor size, mitotic index, and biomarkers Ki67, and Bcl-2 identify two groups with different prognosis. Gynecol. Oncol. 121: 328-333. http:// dx.doi.org/10.1016/j.ygyno.2011.01.022

DerSimonian R and Kacker R (2007). Random-effects model for meta-analysis of clinical trials: an update. Contemp. Clin. Trials 28: 105-114. http://dx.doi.org/10.1016/j.cct.2006.04.004

Egger M and Smith GD (1998). Bias in location and selection of studies. BMJ 316: 61-66. http://dx.doi.org/10.1136/ bmj.316.7124.61

Gannon BR, Manduch M and Childs TJ (2008). Differential Immunoreactivity of p16 in leiomyosarcomas and leiomyoma variants. Int. J. Gynecol. Pathol. 27: 68-73. http://dx.doi.org/10.1097/pgp.0b013e3180ca954f

Hakverdi S, Güngören A, Yaldiz M, Hakverdi AU, et al. (2011). Immunohistochemical analysis of p16 expression in uterine smooth muscle tumors. Eur. J. Gynaecol. Oncol. 32: 513-515.

Genetics and Molecular Research 16 (1): gmr16019481 
Hellman K, Lindquist D, Ranhem C, Wilander E, et al. (2014). Human papillomavirus, p16(INK4A), and Ki-67 in relation to clinicopathological variables and survival in primary carcinoma of the vagina. Br. J. Cancer 110: 1561-1570. http://dx.doi.org/10.1038/bjc.2014.32

Hendrickson MR, Tavassoli FA, Kempson RL, Mc Cluggage WG, et al. (2003). Mesenchymal tumours and related lesions. In: World Health Organization Classification of Tumours. Pathology and Genetics of Tumours of the Breast and Female Genital Organs. (Tavassoli FA and Devilee P, eds.). IARC Press, Lyon, France, 233-244.

Huang K, Li LA, Meng YG and Fu XY (2014). p16 expression in patients with cervical cancer and its prognostic significance: meta-analysis of published literature. Eur. J. Obstet. Gynecol. Reprod. Biol. 183: 64-69. http://dx.doi. org/10.1016/j.ejogrb.2014.10.016

Huang T, Chen X, Hong Q, Deng Z, et al. (2015). Meta-analyses of gene methylation and smoking behavior in non-small cell lung cancer patients. Sci. Rep. 5: 8897. http://dx.doi.org/10.1038/srep08897

Ip PP, Cheung AN and Clement PB (2009). Uterine smooth muscle tumors of uncertain malignant potential (STUMP): a clinicopathologic analysis of 16 cases. Am. J. Surg. Pathol. 33: 992-1005. http://dx.doi.org/10.1097/ PAS.0b013e3181a02d1c

Liang Y, Zhang X, Chen X and Lü W (2015). Diagnostic value of progesterone receptor, p16, p53 and pHH3 expression in uterine atypical leiomyoma. Int. J. Clin. Exp. Pathol. 8: 7196-7202.

Mills AM, Ly A, Balzer BL, Hendrickson MR, et al. (2013). Cell cycle regulatory markers in uterine atypical leiomyoma and leiomyosarcoma: immunohistochemical study of 68 cases with clinical follow-up. Am. J. Surg. Pathol. 37: 634642. http://dx.doi.org/10.1097/PAS.0b013e318287779c

Moravek MB, Yin P, Ono M, Coon JS, 5th, et al. (2015). Ovarian steroids, stem cells and uterine leiomyoma: therapeutic implications. Hum. Reprod. Update 21: 1-12. http://dx.doi.org/10.1093/humupd/dmu048

Ndiaye C, Mena M, Alemany L, Arbyn M, et al. (2014). HPV DNA, E6/E7 mRNA, and p16INK4a detection in head and neck cancers: a systematic review and meta-analysis. Lancet Oncol. 15: 1319-1331. http://dx.doi.org/10.1016/ $\underline{\text { S1470-2045(14)70471-1 }}$

O'Neill CJ, McBride HA, Connolly LE and McCluggage WG (2007). Uterine leiomyosarcomas are characterized by high p16, p53 and MIB1 expression in comparison with usual leiomyomas, leiomyoma variants and smooth muscle tumours of uncertain malignant potential. Histopathology 50: 851-858. http://dx.doi.org/10.1111/j.13652559.2007.02699.x

Ortega S, Malumbres M and Barbacid M (2002). Cyclin D-dependent kinases, INK4 inhibitors and cancer. Biochim. Biophys. Acta 1602: 73-87.

Peters WA, 3rd, Howard DR, Andersen WA and Figge DC (1994). Uterine smooth-muscle tumors of uncertain malignant potential. Obstet. Gynecol. 83: 1015-1020. http://dx.doi.org/10.1097/00006250-199406000-00023

Ruas M and Peters G (1998). The p16INK4a/CDKN2A tumor suppressor and its relatives. Biochim. Biophys. Acta 1378: F115-F177.

Sano T, Oyama T, Kashiwabara K, Fukuda T, et al. (1998). Immunohistochemical overexpression of p16 protein associated with intact retinoblastoma protein expression in cervical cancer and cervical intraepithelial neoplasia. Pathol. Int. 48: 580-585. http://dx.doi.org/10.1111/j.1440-1827.1998.tb03954.x

Slatter TL, Hsia H, Samaranayaka A, Sykes P, et al. (2015). Loss of ATRX and DAXX expression identifies poor prognosis for smooth muscle tumours of uncertain malignant potential and early stage uterine leiomyosarcoma. J Pathol Clin Res 1: 95-105. http://dx.doi.org/10.1002/cjp2.11

Tavassoli FA and Devilee P (2003). World Health Organization Classification of Tumours: Tumours of the Breast and Female Genital Organs. (Tavassoli FA and Devilee P, eds.). IARC Press, Lyon, France, 236-239.

Ünver NU, Acikalin MF, Öner Ü, Ciftci E, et al. (2011). Differential expression of P16 and P21 in benign and malignant uterine smooth muscle tumors. Arch. Gynecol. Obstet. 284: 483-490. http://dx.doi.org/10.1007/s00404-010-1690-Z

Yanai H, Wani Y, Notohara K, Takada S, et al. (2010). Uterine leiomyosarcoma arising in leiomyoma: clinicopathological study of four cases and literature review. Pathol. Int. 60: 506-509. http://dx.doi.org/10.1111/j.1440-1827.2010.02549.x

Zaloudek CJ and Hendrickson CJ (2002). Mesenchymal tumors of the uterus. In: Blaustein's pathology of the female genital tract. (Kurman RJ, ed.). Springer, New York, USA, 561-615.

Zong L, Chen P, Jiang J, Wang H, et al. (2012). Correlation between p16 expression and malignant risk of gastrointestinal stromal tumor: evidence from nine studies. Hepatogastroenterology 59: 1458-1463.

Genetics and Molecular Research 16 (1): gmr16019481 